\title{
Análisis de protección para casco de ciclista sometido a impacto virtual por método de elemento finito
}

\author{
Protection analysis for cyclist helmet under virtual impact by \\ finite element method
}

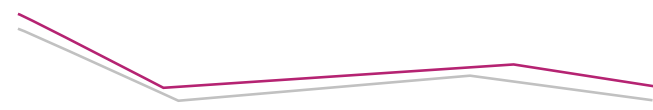

Eddie Nahúm Armendáriz Mireles', José Adalberto Castillo Robles', Marco Antonio López Gutiérrez', Enrique Rocha Rangel ${ }^{1 *}$

\begin{abstract}
Armendáriz Mireles, E. N., Castillo Robles, J. A., López Gutiérrez, M. A., Rocha Rangel, E. Análisis de protección para casco de ciclista sometido a impacto virtual por método de elemento finito. Investigación y Ciencia de la Universidad Autónoma de Aquascalientes. Número 70: 44-49, enero-abril 2017.
\end{abstract}

RESUMEN the finite element method; the entire methodology of this work was based on the Snell standards. The per-

El uso de la bicicleta implica un gran riesgo de sufrir lesiones por caídas, dentro de las cuales las de mayor riesgo son las lesiones en la cabeza, por lo cual es indispensable el uso de equipo de protección. El presente proyecto se enfoca en el análisis de la protección ejercida por un casco de ciclista realizado a través del método de elemento finito, la metodología se basó en las normas Snell. Se determinaron las tensiones que absorbe el casco, se simuló un impacto frontal variando el grosor del material absorbente del casco (30 mm, $20 \mathrm{~mm}$ y $10 \mathrm{~mm}$ ), el cual protege una esfera de aluminio sólida de $4.7 \mathrm{~kg}$, que representaba la cabeza de un ciclista. Los resultados permiten concluir que un casco para ciclista es capaz de absorber más de $90 \%$ de las tensiones y mejora el nivel de protección como se observa en el caso de estudio realizado con el grosor de $20 \mathrm{~mm}$.

\section{ABSTRACT}

Bikes involve great risk of injury from falls; among the major risks are head injuries. Therefore, it is essential to use protective elements. This project focuses on a study to analyze protection for a helmet for cyclists using

Palabras clave: casco de ciclista, análisis de esfuerzos, prueba de impacto, simulación, seguridad deportiva.

Keywords: bicycle helmet, stress analysis, impact test, simulation, sports security. centage of absorbed tension was determined by simulating a frontal impact by varying the thickness of the material of the helmet $(30 \mathrm{~mm}, 20 \mathrm{~mm}$ and $10 \mathrm{~mm}$ ) that protects a $4.7 \mathrm{~kg}$ sphere of solid aluminum, emulating the head of a bicyclist. From the results it was determined that the helmet can absorb more than $90 \%$ of the tensions, the helmet with $20 \mathrm{~mm}$ of thickness presents the best results.

\section{INTRODUCCIÓN}

Las lesiones en la cabeza han generado una mayor preocupación en algunos deportes, especialmente en los de contacto. Los cascos han sido adaptados como dispositivos de prevención y protección de lesiones en la cabeza (Luo y Liang, 2013). Los mismos no son legalmente obligatorios en varios países pero son muy convenientes. El casco debe ajustarse bien a la cabeza, colocarse a la altura de las cejas y nunca inclinado hacia la parte posterior de la misma (Roberts, 2012). En un accidente de bicicleta, las lesiones cerebrales pueden ser penetrante 0 fracturada y no penetrante y no fracturada. Los dispositivos de protección, en este caso los cascos, pueden proteger eficazmente contra la lesión penetrante, pero pueden tener un efecto mucho menor sobre la lesión no penetrante, dado que el cerebro puede ser lesionado en un impacto dejando el cráneo esencialmente intacto.

El mecanismo de la lesión no penetrante es mucho más complicado que el de la penetrante. Este tipo de lesiones está determinado por la complejidad y la estructura anatómica fina del cerebro humano, 


\section{IIVESTIGAGIÓn Y CUERCIA DE LA UחIVERSIDAD AUTÓNOMA DE RGUASCHLIETTES}

aunque a la fecha no todos los mecanismos de la lesión cerebral se conocen bien (Luo et al., 2008).

Cuando la cabeza hace impacto repentino y violento con un objeto rígido, la pequeña parte de la cabeza que es golpeada deja de moverse inmediatamente, pero el resto sigue en movimiento (Snell Memorial Foundation, 2015), lo que puede provocar lesiones irreversibles. El uso de un casco certificado puede reducir hasta en $50 \%$ la mortalidad en un percance. Lamentablemente en México solo se tienen datos de estos elementos en cinco entidades y son de motocicleta (Sánchez, 2015). Aunque el uso del casco de bicicleta ha demostrado poder reducir lesiones en la cabeza de manera significativa, ningún casco puede proteger al usuario de todos los accidentes previsibles. Por tanto, lesión, muerte o daño permanente pueden ocurrir en accidentes que exceden la capacidad de protección de cualquier casco, incluso los que cumplan los requisitos de las normas establecidas (Chang et al., 2003).

Los cascos se construyen de modo que la energía de un golpe es administrada por el mismo, lo que puede provocar su destrucción parcial o total. Debido a que el daño puede no ser fácilmente evidente, se recomienda que un casco impactado en un accidente sea devuelto al fabricante para su inspección completa. Si no es posible hacerlo, siempre debe ser destruido y reemplazado (Neumayr, 2004; Sánchez, 2015).

La Figura 1 muestra el armazón de plástico exterior y los componentes principales del casco, según su diseño, agrega capacidad de distribución de cargas e impide que objetos penetren en él. El cuerpo principal del casco tiene agujeros de ventilación y está hecho de material ligero que distribuye y absorbe la energía de un impacto.

Durante un impacto como se muestra en la Figura 2, (a) se resalta el daño al cerebro y al cráneo al no usar casco de protección, por su parte la imagen (b) muestra cómo el material absorbe y permite que el cerebro desacelere de forma que se disminuya el daño al impactar, la cabeza hace presión sobre el casco, el cerebro gana con esto un poco más de tiempo y distancia adicional para disminuir la velocidad. El casco ejerce fuerza para disminuir la velocidad de la cabeza hasta frenarla de forma más suave a la que sería de no tenerse el casco (Snell Memorial Fundación, 2015).

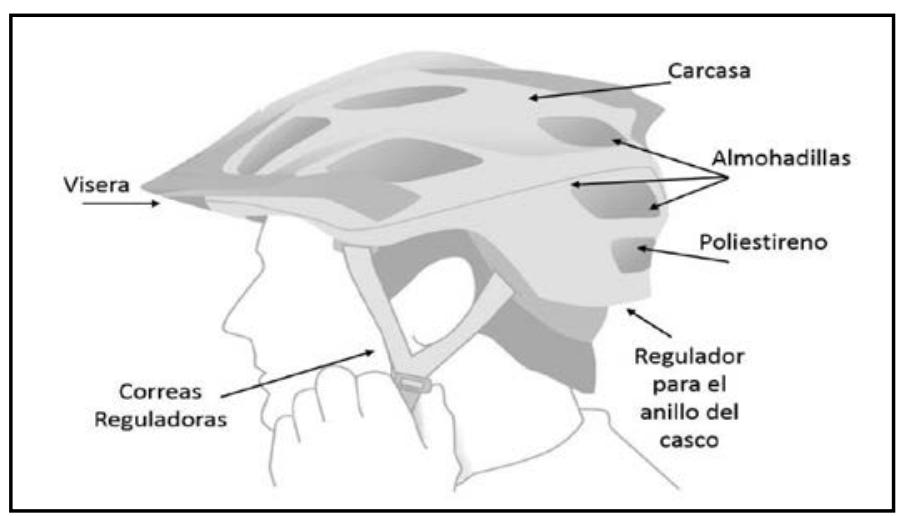

Figura 1. Componentes principales de un casco estándar de ciclista. Imagen tomada de tatoo.ws. Editada por Eddie Armendáriz.

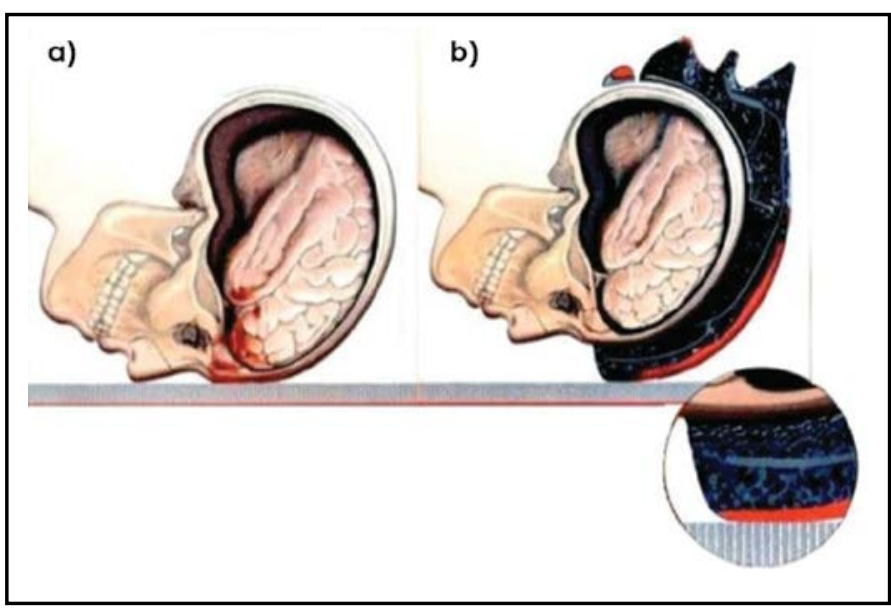

Figura 2. Vista seccionada de la cabeza y cerebro mientras sufre una caída frontal. Imagen tomada de bizirutas.com.

La capacidad de protección puede disminuir con el tiempo. Algunos cascos están hechos de materiales que se deterioran $y$, por tanto, tienen una vida útil limitada. En la actualidad se recomienda que los de bicicleta puedan ser reemplazados a los 5 años de uso o antes si están deteriorados. El material ideal de acuerdo a las normas Snell debe ser durable, resistente a la exposición al sol, lluvia, polvo y no degradable a temperaturas extremas en almacenaje y transportación. Además, debe soportar vibraciones, sudor y productos usados en cabello y piel (Snell Memorial Foundation, 1990). El poliestireno expandido de alta densidad cumple totalmente los requisitos de las normas Snell, por lo que es el material más utilizado a nivel comercial realiza un análisis del desempeño en la protección contra lesiones craneales con el uso de casco para ciclistas sometido a pruebas de impacto mediante el programa informático de simulación de esfuerzos por en cascos para ciclistas. En el presente estudio se 


\section{IIVESTIGAGIÓก Y CIECEIA DE LA UחIVERSIDAD AUTÓกOMA DE RGUASCALIERTES}

elemento finito (Nieto Miranda, 2005), evaluando el comportamiento en los esfuerzos conforme se hace variar el grosor del material sometido a pruebas de impacto. Una ilustración de esto se puede observar en la Figura 3.

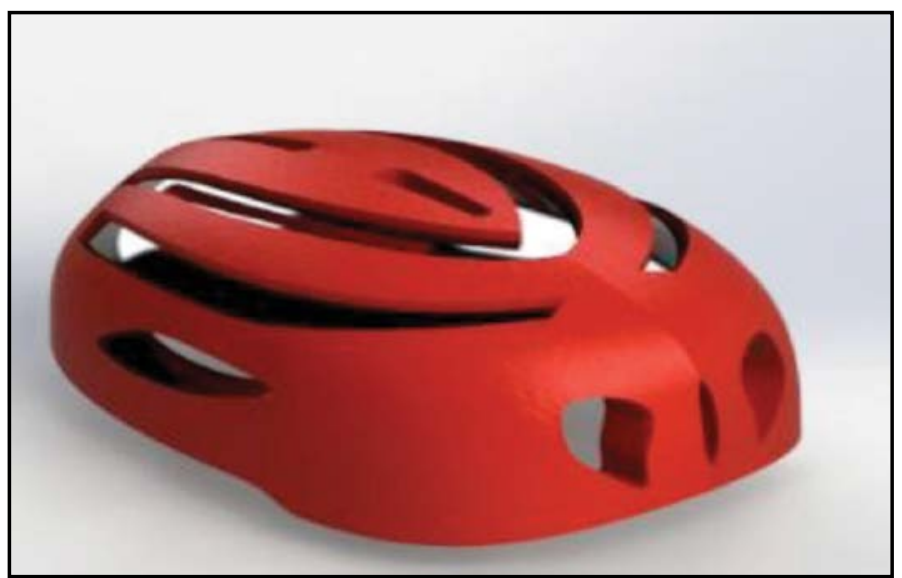

Figura 3. Diseño propuesto. Imagen elaborada por Adalberto Castillo.

\section{MATERIALES Y MÉTODOS}

En este trabajo se analizó el diseño de un casco hecho de poliestireno expandido de alta densidad con tres diferentes espesores $(10 \mathrm{~mm}, 20 \mathrm{~mm}$ y 30 $\mathrm{mm}$ ), para ello se hizo uso de la esfera sola y del casco sobre la esfera. En total se elaboraron cinco estudios. A cada modelo se le realizó un estudio de impacto frontal utilizando el programa de cómputo Solidworks Simulation (Dassault Sistems) que consiste en un entorno virtual basado en elemento finito, esta herramienta de diseño permite predecir el comportamiento de prototipos o piezas sujetas a pruebas con un nivel de aproximación fiable (Gómez González, 2008; Gaff et al., 2015). Las condiciones para estas pruebas fueron de impacto frontal contra una superficie plana rígida a una velocidad de $8.33 \mathrm{~m} / \mathrm{s}$, que equivalen a la velocidad media de los ciclistas de ultramaratones (Baker et al., 1993), con una esfera de aluminio sólida de $4.75 \mathrm{~kg}$ (Chang et al., 2003; Mills y Gilchrist, 2008) y los tres espesores antes indicados, como se muestra en la Tabla 1.

Durante la simulación se utilizó la esfera de aluminio sólida, así como una sección de la parte frontal del casco con las características reportadas en los trabajos realizados por Mills y Gilchrist (1991). Se utilizó un mallado sólido basado en curvatura con elementos cuadráticos de alto orden de cuatro puntos jacobianos simulando el comportamiento que tendría al ser usado por una persona (Figura 4), con los esfuerzos en tensiones Von Mises (TVM), proporcionando los parámetros de nivel de protección con el uso de casco para ciclistas, como se detalla en el trabajo de Mills y Gilchrist (2008).

\section{RESULTADOS}

\section{Estudio de impacto con una esfera}

La Figura 5 muestra la zona de impacto y la

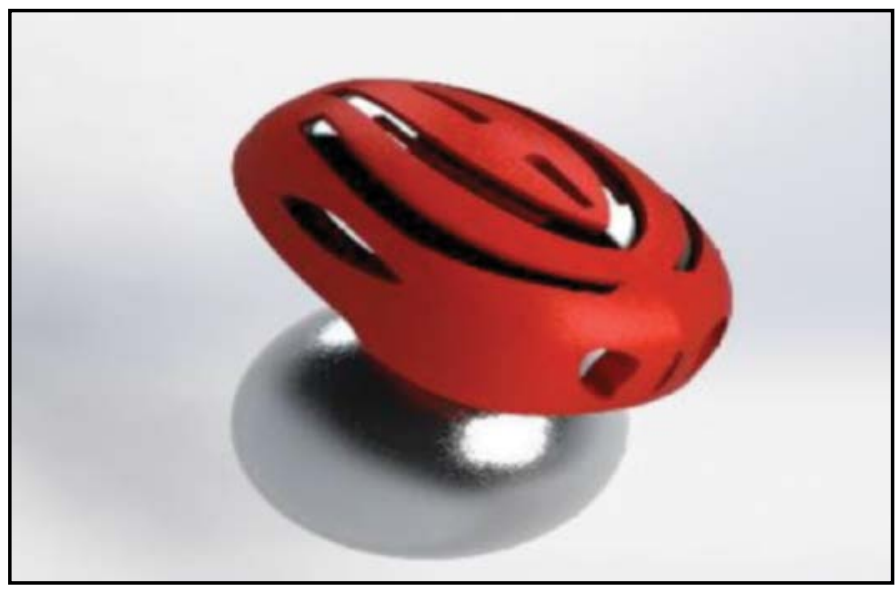

Figura 4. Casco rodeando a la esfera por la parte frontal. Imagen elaborada por Adalberto Castillo.

Tabla 1. Parámetros utilizados en los estudios realizados a la esfera y a la sección frontal del casco que rodea a la esfera sólida de aluminio

\begin{tabular}{|c|c|c|c|c|}
\hline Estudio & Espesor & $\begin{array}{c}\text { Esfera de Al } \\
\text { (diámetro } \mathbf{~ m m} \text { ) }\end{array}$ & $\begin{array}{c}\text { Aceleración } \\
\left(\mathbf{m} / \mathbf{s}^{\mathbf{2}}\right)\end{array}$ & $\begin{array}{c}\text { Velocidad } \\
(\mathbf{m} / \mathbf{s})\end{array}$ \\
\hline 1 & No & 150 & 9.81 & 8.33 \\
\hline 2 & 10 & 150 & 9.81 & 8.33 \\
\hline 3 & 20 & 150 & 9.81 & 8.33 \\
\hline 4 & 30 & 150 & 9.81 & 8.33 \\
\hline 5 & 20 & 150 & 9.81 & 4.15 \\
\hline
\end{tabular}




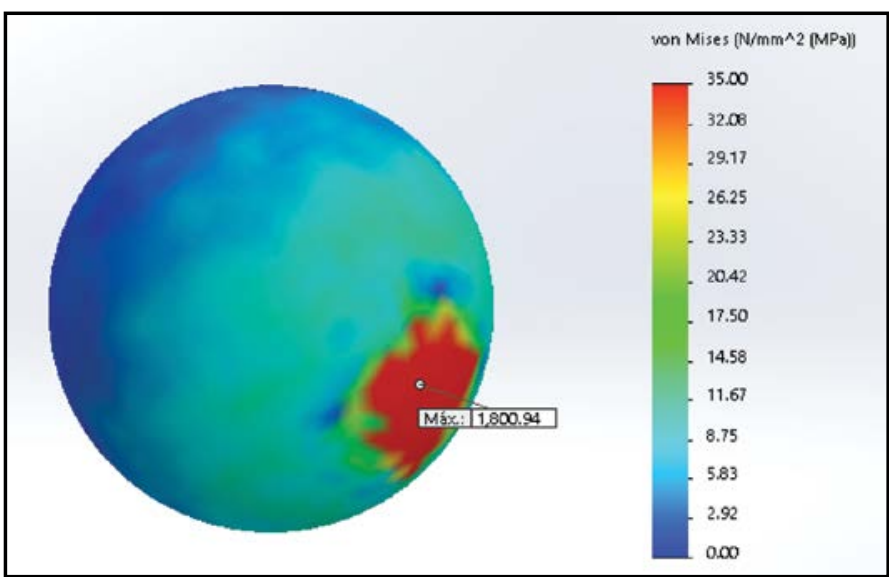

Figura 5. Resultantes del estudio de impacto frontal realizado a la esfera de aluminio sólida. Imagen elaborada por Adalberto Castillo.

distribución de los esfuerzos Von Mises mediante escala de colores, así como una nota con el nodo de máximo valor del estudio de 1,800 Mpa, sobrepasando el límite elástico del material.

\section{Estudio de impacto con dos esferas y casco de $10 \mathrm{~mm}$} A diferencia del estudio anterior, en este ensayo se aumenta el casco con espesor de $10 \mathrm{~mm}$ como se observa en la Figura 6, al concluir la simulación el casco se muestra separado de la esfera para observar la zona de impacto. Los valores máximos se resaltan en ambos objetos, de los cuales el casco registró el mayor valor de tensiones Von Mises 175.16 Mpa en la zona de impacto. Por su parte, la esfera presenta un valor máximo de 164.3 Mpa.

Estudio de impacto con tres esferas y casco de $20 \mathrm{~mm}$ En la tercera prueba se trabaja con un espesor del

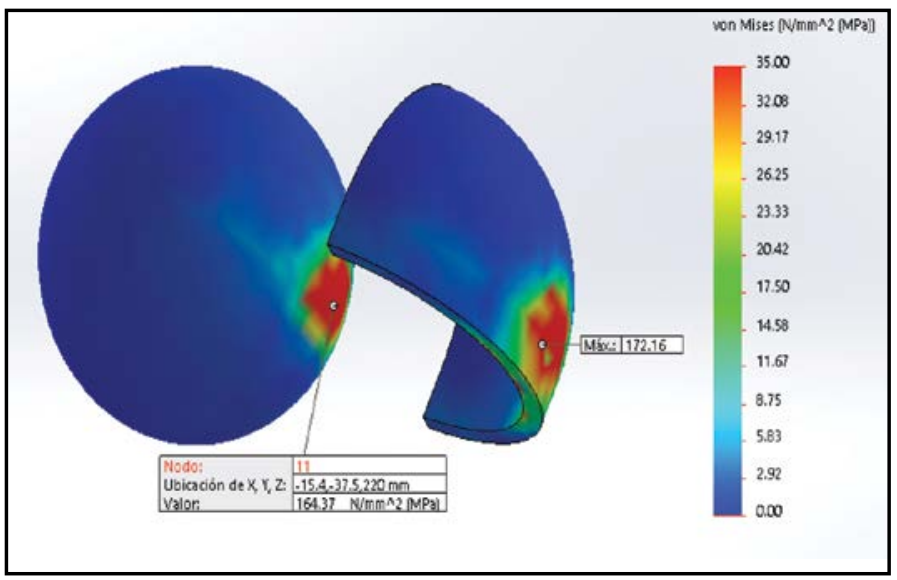

Figura 6. Imagen del estudio de impacto frontal realizado a la esfera de aluminio usando el casco de protección con grosor de $10 \mathrm{~mm}$. Imagen elaborada por Adalberto Castillo. casco de $20 \mathrm{~mm}, 100 \%$ mayor que el anterior. De las imágenes obtenidas, en la Figura 7 se observa que presentó un valor máximo en tensiones Von Mises de $92.1 \mathrm{Mpa}$ y la esfera alcanzó su máximo valor de $68 \mathrm{Mpa}$ en el mismo nodo (nodo 11) que la prueba anterior.

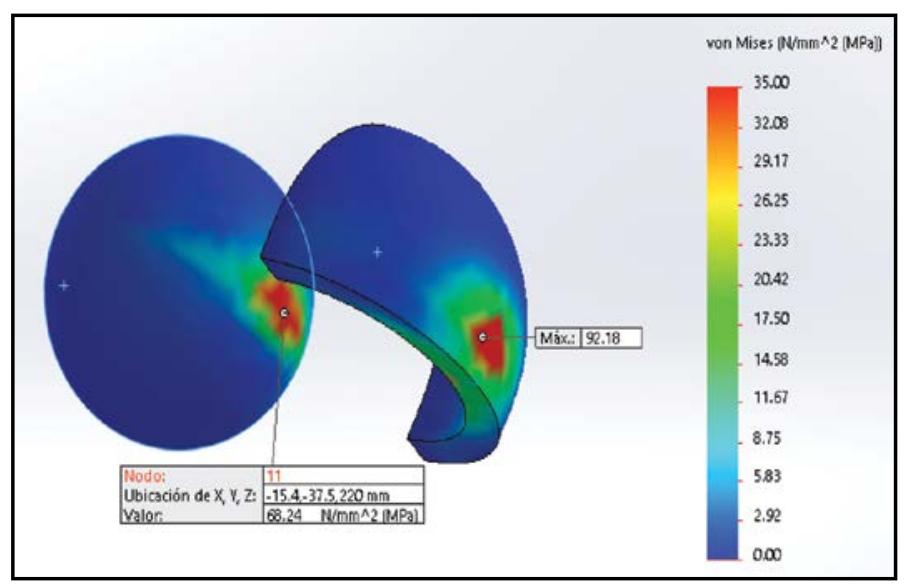

Figura 7. Imagen del estudio de impacto frontal realizado a la esfera de aluminio usando el casco de protección con grosor de 20 mm. Imagen elaborada por Adalberto Castillo.

\section{Estudio de impacto con cuatro esferas y casco de 30 $\mathrm{mm}$}

En la simulación de impacto para el casco con grosor de $30 \mathrm{~mm}$ (Figura 8), se observa en la superficie de contacto el valor máximo de tensiones Von Mises de 63.7 Mpa, el cual es el menor en comparación con los estudios anteriores. Por su lado, la esfera presenta un valor de 43.7 Mpa en TVM, el cual es $25 \mathrm{Mpa}$ menos que el espesor anterior. Los datos mostrados en los estudios colocan como menor valor

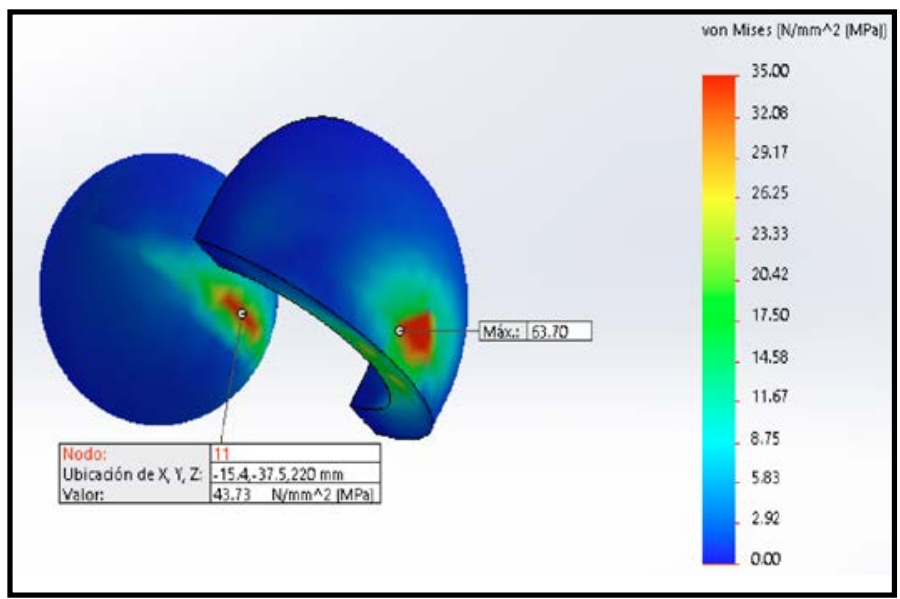

Figura 8. Imagen del estudio de impacto frontal realizado a la esfera de aluminio usando el casco de protección con grosor de $30 \mathrm{~mm}$. Imagen elaborada por Adalberto Castillo. 
IIVESTIGACIÓn Y CIERCIA DE LA UNIVERSIDAD AUTÓNOMA

de tensiones Von Mises máximo a la esfera que usa el casco con espesor de $30 \mathrm{~mm}$.

\section{Estudio de impacto con cinco esferas y casco de 20 $\mathrm{mm}$}

La Figura 9 muestra los resultados del estudio donde se disminuyó la velocidad de impacto a $4.15 \mathrm{~m} / \mathrm{s}$, que corresponde a la velocidad promedio de un ciclista en medio urbano (15 km/h), con el fin de observar el comportamiento bajo estas condiciones. Los valores máximos arrojados por este estudio son: en el casco $44 \mathrm{Mpa}$ y en la esfera 26.7 Mpa en tensión Von Mises. Estos valores revelan que el esfuerzo recibido en la esfera está por debajo del límite elástico del material utilizado.

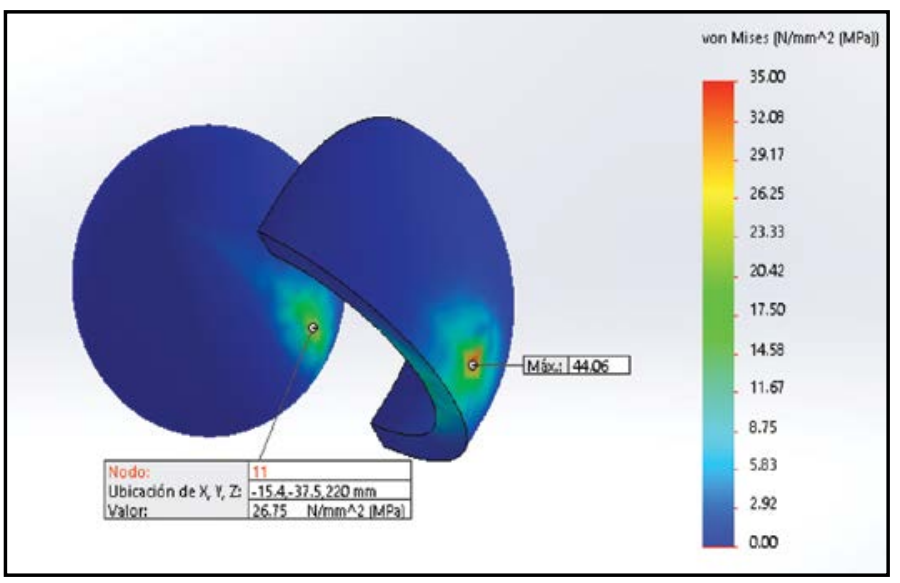

Figura 9. La imagen muestra la esfera de aluminio y el casco con grosor de $20 \mathrm{~mm}$ después de un impacto a $4.15 \mathrm{~m} / \mathrm{s}$ (15 km/h). Imagen elaborada por Adalberto Castillo.

\section{DISCUSIÓN}

Los elementos utilizados variaron ampliamente entre los diferentes análisis realizados. En el estudio uno de la esfera sola se observaron valores que indican un fallo en el objeto debido a que se rebasó su límite elástico (35 Mpa) significativamente. En cuanto al estudio dos, el máximo valor de TVM observado en comparación con la prueba uno es claramente menor, lo cual demuestra el efecto y capacidad de protección posible al utilizar estos dispositivos.

En los estudios tres y cuatro se observa una mejora en su desempeño, puesto que se reducen los valores de tensión por debajo de la mitad de los registrados con la esfera de aluminio en el estudio uno. Sin embargo, el límite elástico del material es rebasado en ambos casos.
Un aspecto a resaltar es la diferencia en las tensiones que presenta la esfera con el de $20 \mathrm{~mm}$ contra el de $10 \mathrm{~mm}$, cerca de los $96 \mathrm{Mpa}$, a su vez mayor que los $25 \mathrm{Mpa}$ que se observan usando el doble de material ( $30 \mathrm{~mm}$ ). Por su parte, en el segundo estudio, en el cual se hace uso del casco con espesor de $10 \mathrm{~mm}$, se presenta un valor máximo en tensiones Von Mises mayor en comparación con los estudios posteriores, lo que lo hace el menos adecuado debido a que absorbe menos energía durante el impacto. Por los datos mostrados en los primeros cuatro estudios se concluye que el que brinda una protección adecuada es el de 20 $\mathrm{mm}$ de espesor, situación que es acorde con lo reportado en la literatura, lo que permite reducir el uso de material en buena parte sin dejar de lado el esfuerzo que soporta uno más robusto que este (Mills y Gilchrist, 1991). Es de resaltar que las condiciones utilizadas para dichas pruebas son a las que pudiera estar expuesto un atleta de ultramaratón; por lo cual queda de manifiesto que el riesgo de sufrir una lesión en la cabeza es alto, aun con el uso de esta protección.

Por otro lado, la quinta prueba se realizó bajo condiciones a las que se expone un ciclista promedio. Dicha prueba sugiere que un casco de protección es capaz de absorber los esfuerzos generados por el impacto. Esto a su vez se traduce en una considerable reducción de posibilidades de sufrir lesiones graves en la cabeza.

De manera general, en este estudio se observa el comportamiento de la esfera de aluminio, donde se resalta la reducción de las tensiones en el punto de impacto cuando se utiliza el de poliestireno de alta densidad. En la Tabla 2 se muestran las tensiones alcanzadas en la esfera y el porcentaje de tensiones que el casco absorbe en los diferentes impactos bajo las mismas condiciones, donde absorbe más de $90 \%$ de la energía del impacto en todos los estudios. Los datos demuestran la diferencia entre grosores, con los cuales se concluye que el grosor del mismo puede estar por debajo de los $30 \mathrm{~mm}$.

CONCLUSIONES

Con la metodología por medio de análisis de elemento finito se logró el objetivo de analizar la capacidad de protección que es posible alcanzar mediante el uso de un casco de ciclista contra lesiones en la cabeza provocadas por impacto frontal. Se concluye que el casco con grosor de 20 
Tabla 2. Resultados de valores máximos de TVM y el porcentaje de reducción de estas debido al grosor del casco

\begin{tabular}{|c|c|c|}
\hline $\begin{array}{c}\text { Grosor del } \\
\text { casco }\end{array}$ & $\begin{array}{c}\text { Tensión en área de } \\
\text { contacto en la esfera }\end{array}$ & $\begin{array}{c}\text { \% de reducción } \\
\text { de tensión }\end{array}$ \\
\hline 0 & 1800 & $0 \%$ \\
\hline 10 & 164,37 & $90 \%$ \\
\hline 20 & 68,24 & $96 \%$ \\
\hline 30 & 43,73 & $97.5 \%$ \\
\hline
\end{tabular}

Elaboración propia.

$\mathrm{mm}$ brinda el mejor desempeño cuando el ciclista se desplaza a una velocidad moderada. El análisis de los esfuerzos se realizó con base en los resultados de tensión Von Mises en una esfera de aluminio sólida. Además se tiene que el casco absorbe una cantidad de energía superior a $90 \%$ de reducción de TVM en todos los casos analizados, suficiente como para poder reducir el daño posible hacia el objeto que protege. El estudio también revela que debe ser desechado una vez que recibió un impacto de esta magnitud, debido a que en ninguno de los casos la esfera quedó libre de esfuerzos. En otras palabras, su función es la de absorber la energía durante un impacto y reducir así las posibilidades de sufrir daños en la cabeza.

\section{LITERATURA CITADA}

- $\quad$ BAKER, S. P. et al. Injuries to bicyclists: a national perspective. Baltimore, MD, US: Johns Hopkins University, p. 91, 1993.

- CHANG, L. T. et al. Finite element analysis of the effect of motorcycle helmet materials against impact velocity. Journal of the Chinese Institute of Engineers, 26(6): 835-843, 2003.

- GAFF, M. et al. Stress Simulation in Layered Wood-Based Materials under Mechanical Loading. Material \& Designs, 87, 1065-1071, 2015.

- GÓMEZ GONZÁlez, S. El gran libro de Solidworks. Editorial Alfaomega Grupo Editor, 2008.

- LUO, Y. y LIANG, Z. Sport helmet design and virtual impact test by imagen-based finite element modeling. 35th Annual Intetnational Conference of the IEEE, EMBC (Engineering in Medicine and Biology Society), Osaka, Japan, 3-7 July, 2013.

- LUO, Y. et al. Recent progress in application of FEM in study of non-penetrating brain injuries. Advances in Theoretical and Applied Mechanics, 1 (225): 240, 2008.

- MILLS, N. J. y GILCHRIST, A. Finite-element analysis of bicycle helmet oblique impacts. International Journal of Impact Engineering, 35(9): 1087-1 101, 2008.

- MILLS, N. J. y GILCHRIST, A. The effectiveness of foams in bicycle and motorcycle helmets. Accident Analysis and Prevention, 23(2): 153-163, 1991.

- NEUMAYR, G. Effect of ultramarathon cycling on the heart rate in elite cyclists. British Journal of Sports Medicine, 38(1): 55-59, 2004.
NIETO MIRANDA, J. J. Análisis de esfuerzos de compresión en el cráneo humano por medio del método del elemento finito. Revista Mexicana de Ingeniería Biomédica, XXVI(1): 16-21, 2005.

- ROBERTS, O. Manual completo de triatlón. 1. España: Hispano europea, p. 34, 2012.

\section{De páginas electrónicas}

- BIZIRUTAS.COM. [Imagen]. Recuperada de http://bizirutas. com/wp-content/uploads/As\%C3\%AD-funciona.jpg

- SÁNCHEZ, J. Ponen su vida en un casco patito. El Universal, Sección Nación, domingo 15 de marzo de 2015. Recuperado el 15 de marzo de 2016, de http://archivo.eluniversal.com. $\mathrm{mx} /$ nacion-mexico/2015/impreso/ponen-su-vidaen-un-cascopatito-224170.html

- SNELL MEMORIAL FOUNDATION. Snell B90A helmet standard. As required by CPSC for helmets worn by children ages 4 years and below, B90C Addendum, 1990. Recuperado de http:// www.smf.org/standards/b/b90astd

- SNELL MEMORIAL FOUNDATION. Use la cabeza y póngase un casco [Tríptico]. North Highlands, CA, US: SNELL MEMORIAL FOUNDATION, 2015. Recuperado el 21 de marzo de 2016, de http://www.smf.org/ssec/b_span_broch.pdf

- TATOO.WS. [Imagen]. Recuperada de https://tatoo.ws/files/ public/tips/cintasajustecasco.jpg 